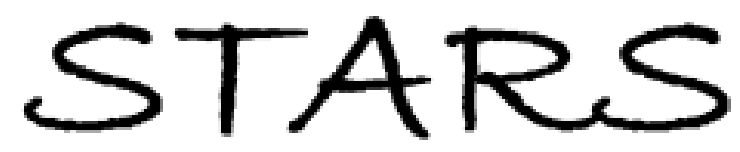

University of Central Florida

STARS

Faculty Bibliography 2010s

Faculty Bibliography

$1-1-2010$

\title{
Image degradation due to scattering effects in two-mirror telescopes
}

James E. Harvey

University of Central Florida

Narak Choi

University of Central Florida

Andrey Krywonos

Gary Peterson

Marilyn Bruner

Find similar works at: https://stars.library.ucf.edu/facultybib2010

University of Central Florida Libraries http://library.ucf.edu

This Article is brought to you for free and open access by the Faculty Bibliography at STARS. It has been accepted for inclusion in Faculty Bibliography 2010 s by an authorized administrator of STARS. For more information, please contactSTARS@ucf.edu.

\section{Recommended Citation}

Harvey, James E.; Choi, Narak; Krywonos, Andrey; Peterson, Gary; and Bruner, Marilyn, "Image degradation due to scattering effects in two-mirror telescopes" (2010). Faculty Bibliography 2010s. 232.

https://stars.library.ucf.edu/facultybib2010/232

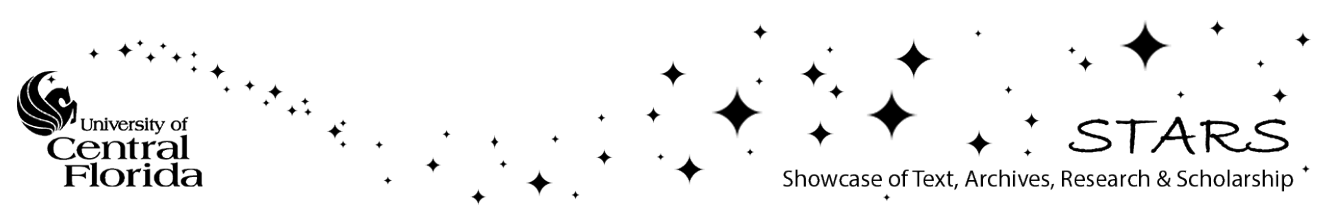




\section{Image degradation due to scattering effects in two-mirror telescopes}

\author{
James E. Harvey, FELLOW SPIE \\ Narak Choi, MEMBER SPIE \\ University of Central Florida \\ College of Optics and Photonics \\ P.O. Box 162700, 4000 Central Florida Boulevard \\ Orlando, Florida 32826 \\ E-mail: harvey@creol.ucf.edu
}

\section{Andrey Krywonos \\ Florida Space Institute \\ Kennedy Space Center, Florida 32899}

\section{Gary Peterson}

Breault Research Organization, Inc.

6400 East Grant Road, Suite 350

Tucson, Arizona 85715

Marilyn Bruner

Bermer Science \& Technology

44 Roosevelt Circle

Palo Alto, California

\begin{abstract}
Image degradation due to scattered radiation is a serious problem in many short-wavelength (x-ray and EUV) imaging systems. Most currently available image analysis codes require the scattering behavior [data on the bidirectional scattering distribution function (BSDF)] as input in order to calculate the image quality from such systems. Predicting image degradation due to scattering effects is typically quite computation-intensive. If using a conventional optical design and analysis code, each geometrically traced ray spawns hundreds of scattered rays randomly distributed and weighted according to the input BSDF. These scattered rays must then be traced through the system to the focal plane using nonsequential ray-tracing techniques. For multielement imaging systems even the scattered rays spawn more scattered rays at each additional surface encountered in the system. In this paper we describe a generalization of Peterson's analytical treatment of in-field stray light in multielement imaging systems. In particular, we remove the smooth-surface limitation that ignores the scattered-scattered radiation, which can be quite large for EUV wavelengths even for state-of-the-art optical surfaces. Predictions of image degradation for a two-mirror EUV telescope with the generalized Peterson model are then numerically validated with the much more computation-intensive ZEMAX ${ }^{\circledR}$ and ASAP ${ }^{\circledR}$ codes. () 2010 Society of Photo-Optical Instrumentation Engineers. [DOI: $10.1117 / 1.3454382]$
\end{abstract}

Subject terms: image degradation; surface scattering; stray light; BSDF; EUV telescopes.

Paper 100144R received Mar. 1, 2010; revised manuscript received Apr. 26, 2010; accepted for publication Apr. 30, 2010; published online Jul. 1, 2010.

\section{Introduction and Overview}

Surface scattering phenomena continue to be an important issue in diverse areas of science and engineering in the twenty-first century. In particular, image degradation due to surface scattering from residual optical fabrication errors remains a serious problem in many short-wavelength (x-ray and EUV) imaging applications.

The purpose of this paper is to describe and numerically validate the generalization of a simple analytical treatment of in-field stray light in multielement imaging systems reported by Peterson in 2004! In the more extensive version of that paper that served as a Master's Report at the Optical Sciences Center at the University of Arizona he emphasized that although optical systems are complex, the distribution of scattered light from their elements is not. The halo of scattered light that surrounds a bright source image is merely the sum of the contributions from each element. Furthermore, the scattered-light irradiance distribution from any one element has the form of that element's bidirectional scattering distribution function (BSDF), and its magnitude and scale depend only on the size of the beam that passes through that element.

Most scattered-light analysis is performed by tracing millions of rays on a computer. However, the analytic formulas in Ref. 1 provide insight and understanding that is

0091-3286/2010/\$25.00 @ 2010 SPIE not readily apparent to the casual user of the conventional brute-force ray-tracing approaches. Thus, intuitive design trades can now be performed, and limits on system performance assessed, without the need for access to, or expertise in, commercially available optical analysis codes.

Peterson's treatment was limited by a smooth-surface assumption that justified ignoring the effects of multiple scattering as the radiation encountered multiple elements in propagating through the optical system. We remove that smooth-surface limitation and include the scatteredscattered radiation resulting from moderately rough surfaces in two-mirror telescopes. The resulting simple analytical model is numerically validated by comparing the results with those predicted by the computation-intensive commercially available ZEMAX ${ }^{\circledR}$ and ASAP ${ }^{\circledR}$ codes.

\section{Review of Analytic Expressions for In-field Stray Radiation}

Making use of the Lagrange invariant of first-order imaging theory and the brightness theorem (conservation of radiance), the scattered irradiance in the focal plane of an imaging system from the $j$ 'th element for an in-field point source has been shown by Peterson 1 to be given by

$E_{\mathrm{s} j}(r)=E_{\mathrm{ent}} \pi(\mathrm{na})^{2} T \frac{s_{\mathrm{ent}}^{2}}{s_{j}^{2}} \operatorname{BSDF}\left(\mathrm{na} \frac{r}{s_{j}}\right)$,

where BSDF is the bidirectional scattering distribution function, $r$ is the radial distance from the point-source geo- 


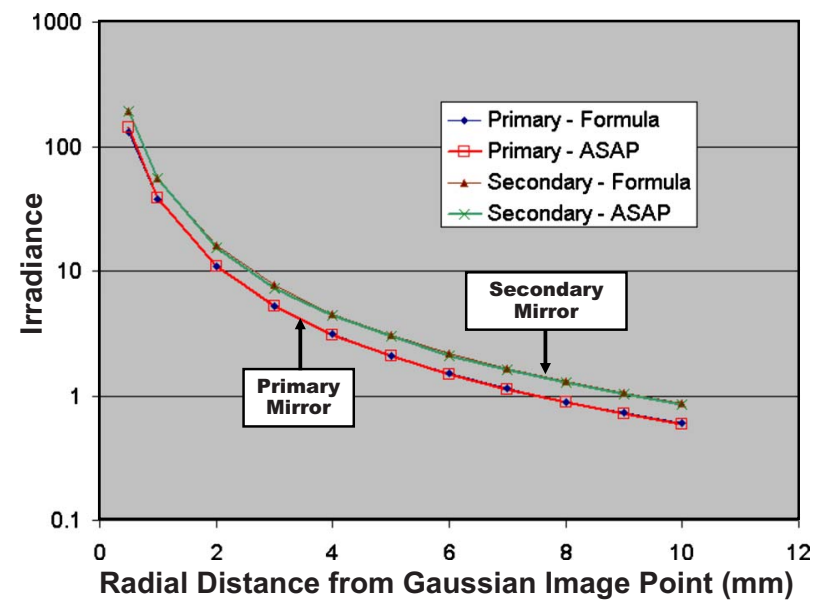

Fig. 1 Numerical validation of the Peterson analytical model for infield stray radiation.

metrical image in the image plane of the telescope, na is the numerical aperture of the system, $T$ is the system transmittance, $s_{\text {ent }}$ is the radius of the entrance pupil, $s_{j}$ is the radius of the beam on the $j$ 'th element, and $E_{\text {ent }}$ is the irradiance in the entrance pupil of the system. This formulation is based on both a smooth-surface and a paraxial assumption.

For a two-mirror telescope, we can thus write

$$
\begin{aligned}
E_{\mathrm{s}}(r)= & E_{\mathrm{ent}} \pi(\mathrm{na})^{2} T s_{\mathrm{ent}}^{2}\left[\frac{\mathrm{BSDF}_{\mathrm{p}}\left(\mathrm{na} r / s_{\mathrm{p}}\right)}{s_{\mathrm{p}}^{2}}\right. \\
& \left.+\frac{\mathrm{BSDF}_{\mathrm{s}}\left(\mathrm{na} r / s_{\mathrm{s}}\right)}{s_{\mathrm{s}}^{2}}\right] .
\end{aligned}
$$

Since $s_{\text {ent }}=s_{\mathrm{p}}$, na $=1 /\left(2 F^{\#}\right)=s_{\mathrm{p}} / f^{\prime}\left(f^{\prime}=\right.$ system focal length $)$, and the total radiant power reaching the focal plane is given by $P_{\mathrm{T}}=E_{\mathrm{ent}} \pi s_{\mathrm{p}}^{2} T$, the scattered irradiance in the telescope focal plane normalized by the total radiant power is given by

$\frac{E_{\mathrm{s}}(r)}{P_{\mathrm{T}}}=\left(\frac{1}{f^{\prime}}\right)^{2}\left[\operatorname{BSDF}_{\mathrm{p}}\left(r / f^{\prime}\right)+\left(\frac{s_{\mathrm{p}}}{s_{\mathrm{s}}}\right)^{2} \operatorname{BSDF}_{\mathrm{s}}\left(\left(s_{\mathrm{p}} / s_{\mathrm{s}}\right)\left(r / f^{\prime}\right)\right)\right]$.

The preceding theoretical expression has been validated numerically with the ASAP code for the case of a Cassegrain telescope whose mirrors exhibit an inverse power-law BSDF described as the two-parameter Harvey model 3 Figure 1 illustrates the irradiance distribution in the focal plane of the telescope from both the primary and the secondary mirrors as predicted by Eq. (3) and compares it with the ASAP scattering calculation (785,000 rays scattered from the primary mirror). The agreement is superb, thus providing a numerical validation of the Peterson analytical model for smooth surfaces and paraxial scattering angles

\section{Generalization of Analytic Treatment for Rough Surfaces}

The fraction of the total reflected radiant power remaining in the specular beam after reflection from a single moderately rough surface is given by 40
$A=\exp \left[-\left(4 \pi \cos \theta_{\mathrm{i}} \sigma_{\text {rel }} / \lambda\right)^{2}\right]$,

and the fraction of the total reflected radiant power contained in the scattered halo, or total integrated scattering (TIS), is given by

$B=\mathrm{TIS}=1-A=1-\exp \left[-\left(4 \pi \cos \theta_{\mathrm{i}} \sigma_{\mathrm{rel}} / \lambda\right)^{2}\right]$,

where $\theta_{\mathrm{i}}$ is the angle of incidence and $\sigma_{\text {rel }}$ is the rms surface roughness measured over the entire range of relevant spatial frequencies (spatial frequencies greater than $1 / \lambda$ are irrelevant since they do not contribute to the scattered radiation) 11 Since Eq. (4) and Eq. (5) are so important to the following discussion, we bring to the attention of the reader that a brief historical perspective of these equations is presented on p. 51 of Ref. 8. Our relevant rms surface roughness, $\sigma_{\text {rel }}$, is the same as the effectiverms surface roughness referred to by Church and Takacs 12 The square of this relevant rms roughness is thus equal to the bandlimited integral of the two-dimensional surface power spectral density (PSD) function integrated out to a spatial frequency of $1 / \lambda$ (for normal incidence), whereas the square of the total, or intrinsic, rms roughness is obtained by integrating the two-dimensional surface PSD from zero to infinity. It should be noted that for two-dimensional surface PSDs exhibiting an inverse power-law behavior at large spatial frequencies, the total, or intrinsic, rms roughness will be infinite (integral of PSD over all spatial frequencies does not converge) if the magnitude of the slope characterizing the power-law behavior is less than 2. However, the relevant rms roughness will always be finite.

\subsection{Scattering in a Two-Mirror Telescope}

For a two-mirror telescope we will have a specular (direct) and a scattered component reflected from the primary mirror. After reflection from the secondary mirror there will be a diminished specular beam (direct-direct component), the scattering function from the primary mirror specularly reflected from the secondary mirror (scattered-direct component), the specularly reflected beam from the primary mirror scattered from the secondary mirror (direct-scattered component), and the scattered radiation from the primary mirror scattered again from the secondary mirror (scattered-scattered component) propagating towards the telescope focal plane as shown in Fig. 2

\subsection{Energy Distribution among Components of the Point Spread Function}

The point spread function (PSF) in the focal plane of the telescope will thus consist of the sum of four components, whose radiant power distributions are as follows:

direct-direct component (specular): $P_{\mathrm{dd}} / P_{\mathrm{T}}=A_{\mathrm{p}} A_{\mathrm{s}}$,

scattered-direct component: $P_{\mathrm{sd}} / P_{\mathrm{T}}=B_{\mathrm{p}} A_{\mathrm{s}}$,

direct-scattered component: $P_{\mathrm{ds}} / P_{\mathrm{T}}=A_{\mathrm{p}} B_{\mathrm{s}}$, 


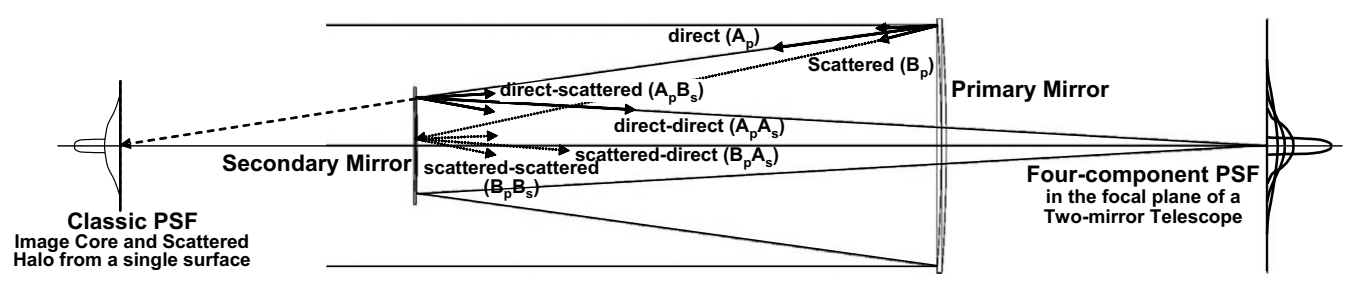

Fig. 2 Illustration of scattering in a two-mirror telescope.

scattered-scattered component: $P_{\mathrm{ss}} / P_{\mathrm{T}}=B_{\mathrm{p}} B_{\mathrm{s}}$.

The quantities $A_{\mathrm{p}}, B_{\mathrm{p}}, A_{\mathrm{s}}$ and $B_{\mathrm{s}}$ are determined from Eq. (4) and Eq. (5) Figure 3 graphically illustrates the radiant power distribution among these four components of the PSF of a two-mirror telescope (same BSDF from both mirrors) as a function of the rms roughness of the mirrors expressed in wavelengths $(\sigma / \lambda)$.

Note that the TIS of the two-mirror telescope is equal to $1-A_{\mathrm{p}} A_{\mathrm{s}}$. It is evident from Fig. 3 that for $\sigma / \lambda<0.02$ scattering effects are modest, with a TIS $<0.12$. However, as $\sigma / \lambda$ increases, the scattered light increases rapidly. At $\sigma / \lambda=0.066$ each of the four components contains $25 \%$ of the total power. As $\sigma / \lambda$ continues to increase, the power in the scattered-scattered component increases and the power in all other components decreases. For $\sigma / \lambda>0.12$ the specular beam has essentially vanished, and for $\sigma / \lambda$ $>0.18$ virtually all of the radiant power is in the scatteredscattered component.

For some short-wavelength applications, such as solar EUV telescopes, surface scattering from state-of-the-art primary and secondary mirrors will dominate both geometrical aberrations and diffraction effects in the degradation of image quality.

\subsection{Calculation of the Scattered-Scattered Irradiance Distribution}

As discussed in the previous subsection, we know the fraction of the total radiant power contained in the scatteredscattered beam. But what is the angular distribution of radiation in the scattered-scattered beam?

Let us think of the scattering process as one whereby each geometrically traced ray, on reflection from a mirror surface, spawns a multitude of scattered rays randomly distributed and weighted according to the mirror's BSDF. These scattered rays are then traced through the system, each one spawning another distribution of scatteredscattered rays when it encounters another mirror surface. Each new family of scattered rays will be randomly distributed and weighted according to the second mirror's BSDF. This is precisely the description of a convolution operation.

We thus generalize the Peterson analytical treatment (viz., remove the smooth-surface limitation) by accurately calculating and adding the effects of the scattered-scattered component to the PSF in the focal plane of the telescope. Assuming isotropic roughness on both the primary and secondary mirrors, we thus construct the following expression:



Fig. 3 Energy distribution among the four PSF components. 
$\operatorname{PSF}(r)=\operatorname{PSF}_{\mathrm{dd}}(r)+\operatorname{PSF}_{\mathrm{sd}}(r)+\operatorname{PSF}_{\mathrm{ds}}(r)+\operatorname{PSF}_{\mathrm{ss}}(r)$.

The first term on the right side of Eq. (10) is given the functional form of the image core, or specular beam, as determined by diffraction and geometrical aberrations. The two middle terms are given the functional form provided by Peterson's analytical expression from Eq. (3) The functional form of the scattered-scattered term is obtained by convolving the two middle terms. In general, this is done by numerically calculating the Hankel transform of the product of the Hankel transforms of the BSDFs provided for the two mirrors. Finally, care is taken to normalize each component of the PSF so that their respective twodimensional integrals (fractional total reflected radiant power) will be equal to $A_{\mathrm{p}} A_{\mathrm{s}}, B_{\mathrm{p}} A_{\mathrm{s}}, A_{\mathrm{p}} B_{\mathrm{s}}$, and $B_{\mathrm{p}} B_{\mathrm{s}}$.

\section{Application to a Two-Mirror EUV Telescope}

We now use the generalized Peterson analytical technique to predict the image quality of a two-mirror RitcheyChretien EUV solar telescope. That allows us to gain insight and understanding, concerning the sometimes nonintuitive surface scattering effects on telescope image quality, that are difficult to obtain with the brute-force computational approaches provided by most of the commercially available optical design and image analysis software. Of course, we numerically validate this generalized Peterson technique by comparing our results with those obtained from the well-known ZEMAX and ASAP optical analysis codes.

We assume a 175-cm-focal-length Ritchey-Chretien telescope design with an aperture diameter of $19 \mathrm{~cm}$ and an obscuration ratio $\varepsilon=0.4$. There will thus be no geometrical aberrations on axis, and the specular beam will be the wellknown Fraunhofer diffraction pattern produced by the annular aperture of the telescope:

$$
\begin{aligned}
\operatorname{PSF}_{\mathrm{dd}}(r)= & \frac{1}{\left(1-\varepsilon^{2}\right)^{2}}\left[\frac{2 J_{1}(x)}{x}-\varepsilon^{2} \frac{2 J_{1}(\varepsilon x)}{\varepsilon x}\right]^{2}, \\
& \text { where } x=\frac{\pi r}{\lambda f / D} .
\end{aligned}
$$

This expression is normalized to a unit volume. It thus needs to be multiplied by the coefficient $A_{\mathrm{p}} A_{\mathrm{s}}$ in the following analysis.

We could merely use a simple Gaussian distribution for the input BSDF to numerically validate our generalization of Peterson's analytical model of irradiance in the focal plane of a two-mirror telescope. However, most optical surfaces fabricated by conventional abrasive grinding and polishing techniques on ordinary amorphous glassy materials tend to result in an inverse power-law surface PSD. We thus use BSDFs predicted by an $\mathrm{ABC}$, or K-correlation, function surface PSD 1112 Figure 4 illustrates such a function fitted to actual surface metrology data from a state-ofthe-art EUV telescope mirror. Four separate metrology instruments were used to measure the optical fabrication errors over the entire range of relevant spatial frequencies.

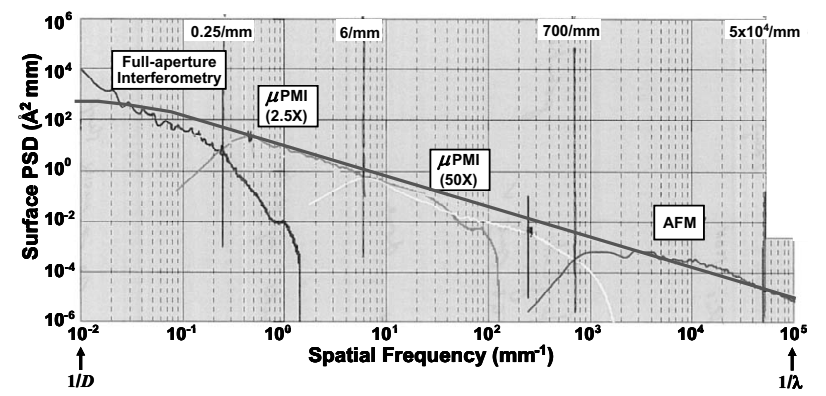

Fig. 4 Composite surface power spectral density (PSD) function determined from four different metrology instruments. An $A B C$, or K-correlation, function has been fitted to the experimental data to characterize the surface over the entire range of relevant spatial frequencies.

\subsection{Predicted Point Spread Function for an $A B C$ Function Surface PSD}

The BSDF profiles illustrated in Fig. 5 have previously been calculated from the metrology data shown in Fig. 4 . The generalized Harvey-Shack surface-scattering theory using an FFTLog algorithm has been used to make such predictions for moderately rough surfaces 11 The three ABC parameters characterizing the surface PSD are indicated in Fig. 5 as are the relevant rms surface roughness and the TIS predicted from Eq. (5) for each of the six wavelengths indicated.

For the shortest wavelength of $93.9 \AA$, the total integrated scattering from a single surface is given by TIS $=0.5650$ and $A=1-\mathrm{TIS}=0.4350$. If we assume that both the primary and the secondary mirror exhibit the same BSDF behavior (indicated in Fig. 5), the respective radiant powers contained in the four components making up the telescope PSF are given by

$$
\begin{aligned}
& A_{\mathrm{p}} A_{\mathrm{s}}=0.1892, \\
& A_{\mathrm{p}} B_{\mathrm{s}}=0.2458, \\
& B_{\mathrm{p}} A_{\mathrm{s}}=0.2458,
\end{aligned}
$$

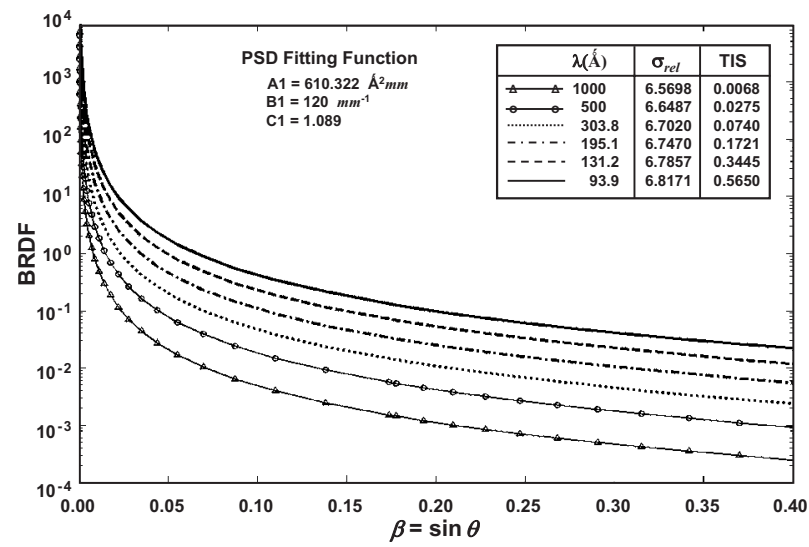

Fig. 5 BSDF profiles for eight different wavelengths, as calculated from actual metrology data from a state-of-the-art EUV telescope mirror. 


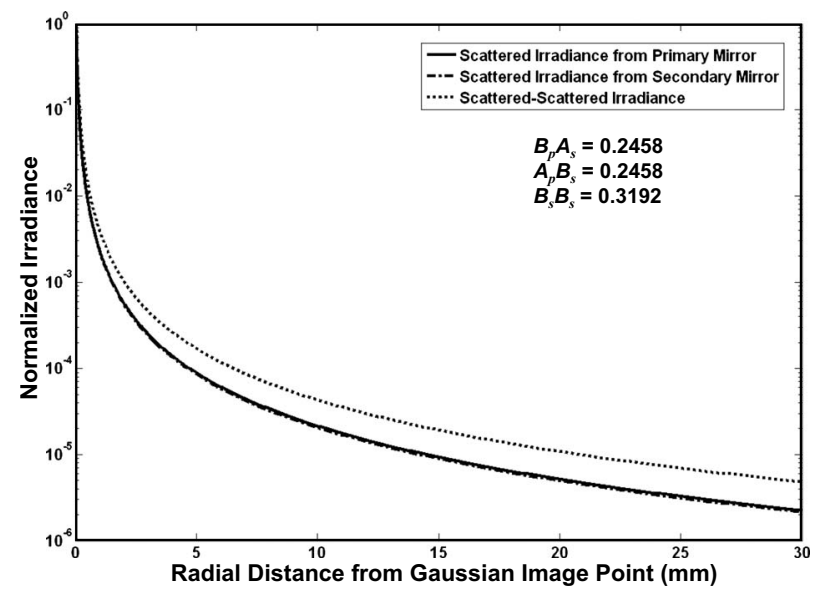

Fig. 6 Radial profiles of the three scattered components contributing to the normalized irradiance distribution (PSF) due to scattering effects at a wavelength $\lambda=93.9 \AA$ in the focal plane of a two-mirror EUV telescope.

$B_{\mathrm{p}} B_{\mathrm{s}}=0.3192$.

It is rather sobering to note that less than $20 \%$ of the total energy reaching the focal plane will reside in the specular beam, or image core. Furthermore, almost $32 \%$ of the energy will reside in the scattered-scattered component. Yet we expect this EUV telescope to provide highresolution images over the entire spectral range $93.9 \AA$ $\leqslant \lambda \leqslant 303.4 \AA$. Clearly there is a strong need to be able to perform accurate predictions of image quality as degraded by surface scattering effects from real metrology data throughout the optical fabrication process 13 We believe that our generalization of Peterson's analytical treatment can substantially aid in that effort.

Noting that $\sin \theta \approx \tan \theta \approx r / f^{\prime}$, the preceding BSDF data are input into Eq. (3) to obtain the scattering functions in the telescope focal plane resulting from the primary and secondary mirror BSDFs individually. The convolution of these two scattering functions provides the form of the scattered-scattered component of the PSF in the telescope focal plane, and the form of the specular (direct-direct) component of the PSF in the telescope focal plane is given by Eq. (11) Profiles of the three scattering functions of the irradiance distribution in the telescope focal plane, properly normalized so that their respective integrals are equal to the coefficients $A_{\mathrm{p}} B_{\mathrm{s}}, B_{\mathrm{p}} A_{\mathrm{s}}$, and $B_{\mathrm{p}} B_{\mathrm{s}}$, are shown in Fig. 6. Note that the scattered-scattered light is indeed the dominant component of the irradiance distribution at this very short EUV wavelength of $93.9 \AA$.

Figure 7 illustrates the total composite PSF for each of the six wavelengths for which BSDFs were provided in Fig. 5 We have tabulated the fraction of the total reflected radiant power in the specular beam and the TIS after reflection from the two telescope mirrors for each of the wavelengths considered. Note that the amount of reflected radiant energy in the specular beam $\left(A_{\mathrm{p}} A_{\mathrm{s}}\right)$ decreases from almost $99 \%$ at $\lambda=1000 \AA$ to less than $19 \%$ at $\lambda=93.9 \AA$. The TIS in the composite PSF thus increases from 0.0136 at $\lambda=1000 \AA$ to 0.8108 at $\lambda=93.9 \AA$.

The apparent irregularities on the PSF profile for longest wavelength of $1000 \AA$ are actually oscillations due to the

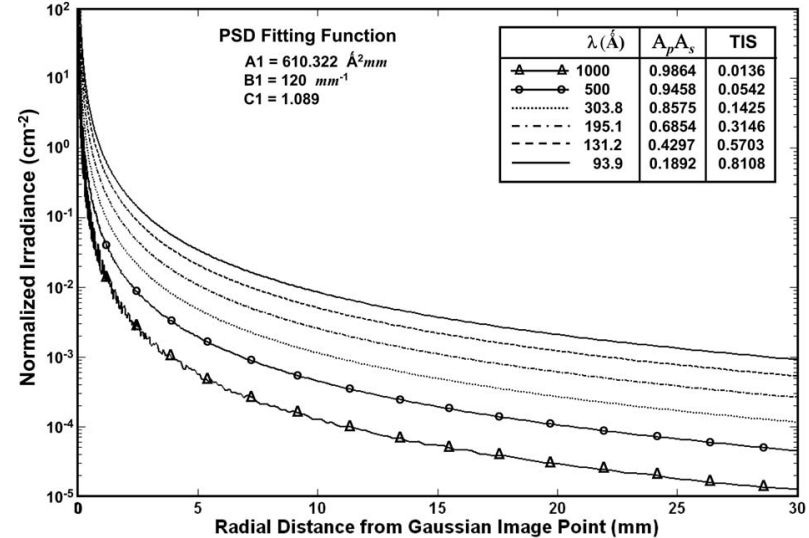

Fig. 7 Radial profiles of the irradiance distribution in the focal plane of the two-mirror EUV telescope due to surface scattering effects from residual optical fabrication errors for the six wavelengths for which BRDFs were supplied in Fig. 4

diffraction rings resulting from Eq. (11) For the shorter wavelengths, the scattered radiation obscures these diffraction rings. Although the relative scattering levels in the wings of the PSFs for the various wavelengths are made apparent in Fig. 7. the relative width of the specular beam is not discernable in these semilog plots.

Perhaps more insight into the distribution of radiant power among the four components of the image in the telescope focal plane is obtained from the log-log plot shown in Fig. 8 for the shortest wavelength of interest (93.9 $\AA$ ). Here we can see that the specular beam decreases as an inverse power law with a slope of -3 , whereas the scattering functions from the primary and the secondary mirrors obey an inverse power law with a slope of approximately -2 (the slope of the two-dimensional surface PSD was -2.089). And the scattered-scattered component is somewhat broader than the two single-scattering functions. Recall that the scattered-scattered beam was obtained by convolving the two singly scattered functions.

Additional insight can be obtained from studying the fractional-encircled-energy plots of the individual compo-



Fig. 8 Log-log plots of the radial profiles of the four components making up the irradiance distribution in the focal plane of the twomirror EUV telescope $(\lambda=93.9 \AA)$. 


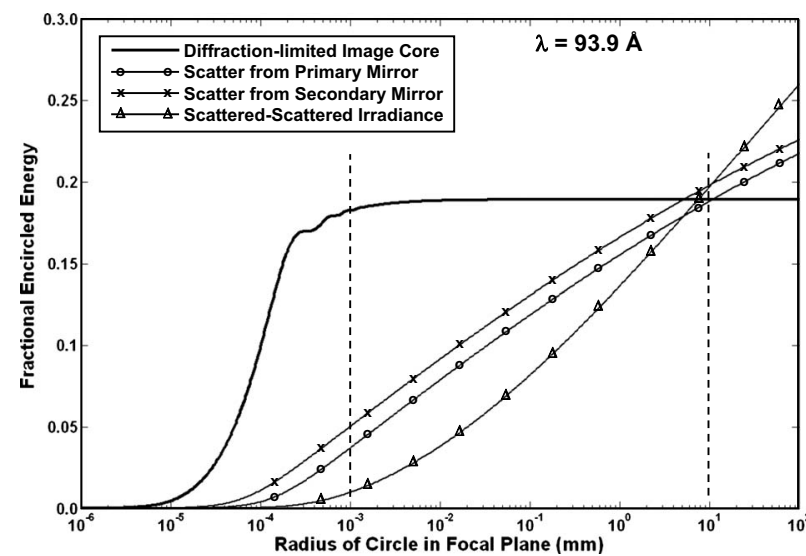

Fig. 9 Fractional-encircled-energy plots of the four individual components making up the PSF in the telescope focal plane.

nents making up the irradiance distribution in the focal plane of the telescope on a linear-log scale, as shown in Fig. 9 for the shortest wavelength of $93.9 \AA$. Note that virtually all of the energy of the diffraction-limited image core is contained within a circle with a radius of $1.0 \mu \mathrm{m}$. It will take a circle radius greater than $10 \mathrm{~mm}$ to include more than $80 \%$ of the total energy in the PSF, and beyond that radius the amount of scattered-scattered light is the dominant component making up the total PSF.

Finally, in Fig. 10 we provide a graph of the fractional encircled energy of the total PSF (projected onto the sky) for a variety of different wavelengths of interest. The angular circle size was obtained by merely rescaling the abscissa by dividing the radial distance in the focal plane by the focal length of the telescope. These plots thus provide an indication of the angular resolution of the EUV telescope. As an example we have highlighted the fraction of the total radiant energy contained in a 20 -arcsec-diameter circle for the wavelengths indicated.

\subsection{Comparison with ZEMAX and ASAP Image Quality Predictions}

Figure 11 gives a direct comparison of the irradiance in the focal plane of a two-mirror EUV telescope as predicted by

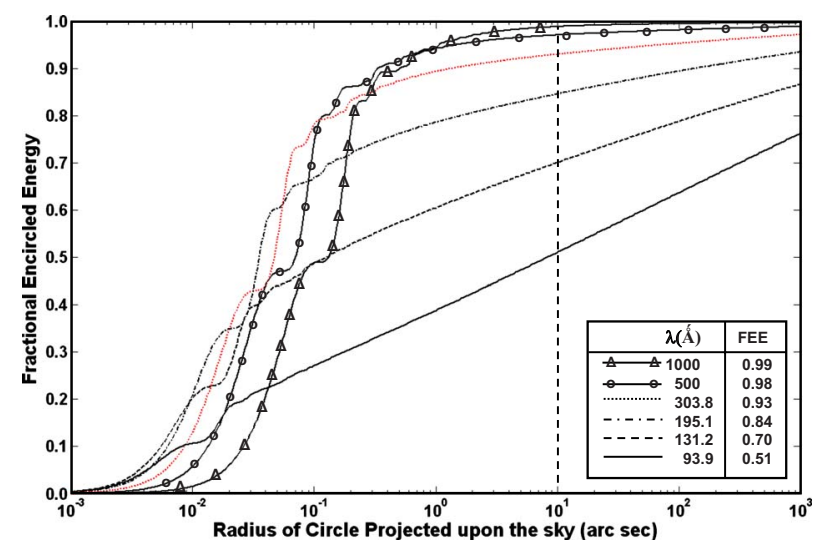

Fig. 10 Fractional encircled energy of the total PSF projected onto the sky. Values for a 20 -arcsec-diameter circle are indicated.

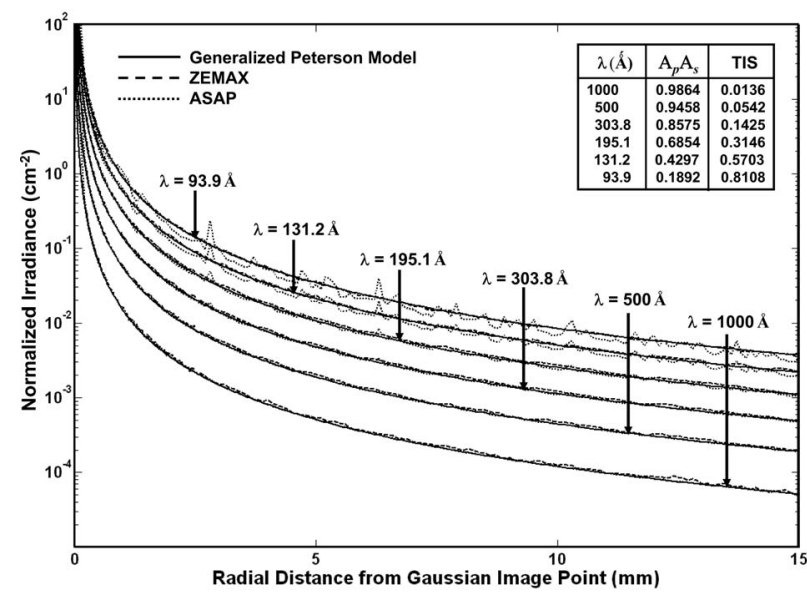

Fig. 11 Comparison of the irradiance distribution in the focal plane of the two-mirror EUV telescope as predicted by the generalized Peterson analytical model, ZEMAX, and ASAP.

our generalized analytical approach with predictions from brute-force scattering calculations from the well-known commercially available ZEMAX and ASAP optical analysis codes. All three approaches used the same design for the two-mirror telescope and applied the BSDF data illustrated in Fig. 4 to both the primary and the secondary mirror. The three approaches are in excellent agreement, even for the shortest wavelength of interest, which does not satisfy the smooth-surface approximation.

\section{Summary and Conclusions}

The ability to quickly and easily perform extensive parametric image quality predictions of the irradiance distribution in the image plane of multielement telescopes exhibiting in-field scattered radiation is invaluable when deriving optical fabrication tolerances necessary to satisfy specific image quality requirements 13 It is particularly valuable for short-wavelength applications where image degradation due to surface scattering is severe. The same parametric image analysis is again useful during the final stages of optical fabrication and testing to make image quality predictions based on actual metrology data.

We have demonstrated that a generalization of Peterson's analytic approach to calculating the irradiance distribution in the focal plane of a multielement imaging system allows one to make accurate image quality predictions even for moderately rough surfaces that do not satisfy the usual smooth-surface requirement. The paraxial limitation is automatically satisfied for practical two-mirror telescope applications. And we have numerically validated that simple analytical approach to making image quality predictions with the computation-intensive calculations provided by the well-known ZEMAX and ASAP codes.

\section{References}

1. G. L. Peterson, "Analytic expressions for in-field scattered light distribution," Proc. SPIE 5178, 184-193 (2004).

2. G. Peterson, "Analytic expressions for in-field stray light irradiance in imaging systems," Master's Report, Optical Sciences Center, Univ. of Arizona (2003).

3. G. Peterson, Stray Light Analysis with ASAP, Advanced ASAP Tutorial, Breault Research Organization (2008). 
4. H. Davies, "The reflection of electromagnetic waves from a rough surface," Proc. I.E.E. Pt. III 101, 209-214 (1954).

5. H. E. Bennett and J. O. Porteus, "Relation between surface roughness and specular reflectance at normal incidence," J. Opt. Soc. Am. 51, 123-129 (1961).

6. P. Beckmann and A. Spizzichino, The Scattering of Electromagnetic Waves from Rough Surfaces, p. 81, Pergamon Press, New York (1963).

7. J. E. Harvey, "Light-scattering characteristics of optical surfaces," PhD Dissertation, Univ. of Arizona (1976)

8. J. M. Bennett and L. Mattsson, Introduction to Surface Roughness and Scattering, Opt. Soc. of Am., Washington (1989).

9. J. C. Stover, Optical Scattering, Measurement and Analysis, 2nd ed., SPIE Optical Engineering Press, Bellingham, WA (1995).

10. J. E. Harvey, C. L. Vernold, A. Krywonos, and P. L. Thompson, "Diffracted radiance: a fundamental quantity in a non-paraxial scalar diffraction theory," Appl. Opt. 38, 6469-6481 (Nov. 1999).

11. J. E. Harvey, N. Choi, A. Krywonos, and J. Marcen, "Calculating BRDFs from surface PSDs for moderately rough surfaces," Proc. SPIE 7426, 74260I (2009)

12. E. L. Church and P. Z. Takacs, "Light scattering from non-Gaussian surfaces," Proc. SPIE 2541, 91-107 (1995).

13. J. E. Harvey, J. Lentz, and J. B. Houston, Jr., "Just-good-enough" optical fabrication," presented at the OSA Top. Mt. on Optical Fabrication and Testing, Rochester, NY (2008).

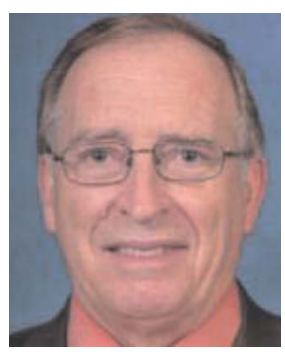

James E. Harvey is an associate professor in the College of Optics and Photonics at the University of Central Florida and a senior staff member of the Center for Research and Education in Optics and Lasers (CREOL). He has a PhD in optical sciences from the University of Arizona and is credited with more than 175 publications and conference presentations in the areas of diffraction theory, surface scattering phenomena, adaptive optics, wavefront sensing, beam sampling technology, optical properties of materials, phased telescope arrays, and x-ray/EUV imaging systems. He is a member of the OSA and a Fellow and past board member of SPIE.

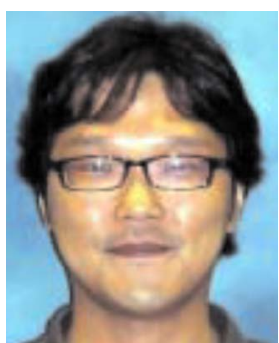

Narak Choi received a bachelor's and a master's degree in physics from Seoul $\mathrm{Na}$ tional University, S. Korea, in 2005 and 2007, respectively. He is currently a PhD student at the Center for Research and Education in Optics and Lasers (CREOL) at the University of Central Florida, doing research on surface scattering theory.

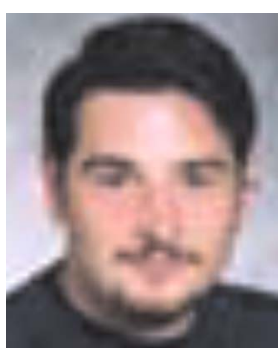

Andrey Krywonos received a bachelor's degree in photonics from SUNY Institute of Technology at Utica, NY, in 1995, and a $\mathrm{PhD}$ degree in optics from the College of Optics and Photonics at the University of Central Florida in 2006. He is currently working with the Florida Spsce Institute at the University of Central Florida in the area of optical design and analysis of spacebased imaging spectrometers.

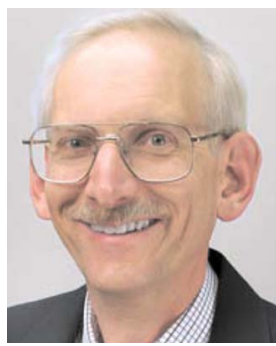

Gary Peterson is a senior optical engineer with Breault Research Organization in Tucson, Arizona. He has a BS in physics from the University of Minnesota, an MS and $\mathrm{PhD}$ in physics from the University of Texas at Dallas, and an MS in optical sciences from the University of Arizona. He has performed stray-light analysis of many spacebased optical systems, including the European Space Agency's MERIS imaging spectrometer, the Infrared Space Observatory, and the XMM-Newton x-ray telescope. He has also designed reflectors and light pipes, and evaluated the luminance and efficiency of projection and backlight systems.

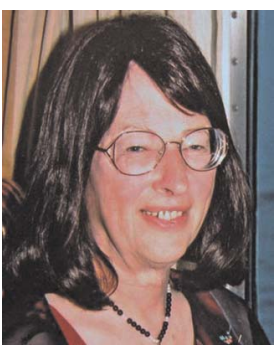

Marilyn Bruner is the CEO of Bermar Science \&Technology LLC of Palo Alto, California. She is a solar physicist with extensive experience in planning and conducting solar research programs from space. She received BS and MS degrees from the University of Arizona in 1957 and 1959, majoring in physics. She received a PhD in physics from the University of Colorado in 1964. Her recent work has concentrated on instrument design with an emphasis on optical design and analysis. 